\title{
Research Paper \\ The Effect of Rose Aromatherapy on Anxiety Before Abdominal Operation
}

\author{
Samane Najafi ${ }^{1}$ (C), Moosa Sajjadi², Amirreza Nasirzadeh ${ }^{3},{ }^{*}$ Hossein Jeddi $^{3}$ ()
}

1. Department of Medical-Surgical Nursing, School of Nursing, Social Development \& Health Promotion Research Center, Gonabad University of Medical Sciences, Gonabad, Iran.

2. Department of Medical-Surgical Nursing, Gonabad University of Medical Sciences, Gonabad, Iran.

3. Student Research Committee, Gonabad University of Medical Sciences, Gonabad, Iran.

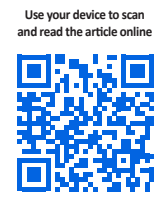

Citration Najafi S, Sajjadi M, Nasirzadeh A, Jeddi H. [The Effect of Rose Aromatherapy on Anxiety Before Abdominal Operation (Persian)]. Quarterly of "The Horizon of Medical Sciences". 2020; 26(2):128-141. https://doi.org/10.32598/hms.26.2.3043.4

doi https://doi.org/10.32598/hms.26.2.3043.4

Key words: Rose, Aromatherapy, Anxiety, Operation

\section{A B STRACT}

Received: 16 May 2019 Accepted: 07 Mar 2020 Available Online: 01 Apr 2020

Aims Preoperative anxiety could increase postoperative pain, the need for analgesics, and the patient's hospital stay. Therefore, this study aimed to evaluate the effect of aromatherapy with rose on preoperative anxiety before abdominal operation.

Methods \& Materials This clinical trial was performed on 90 patients undergoing abdominal operation at 15Khordad Hospital, in 2017. The study patients were assigned to the intervention and control groups by permuted block randomization method ( $n=45 /$ group). The study instrument was the Spielberger StateTrait Anxiety Inventory (STAI). The STAI was completed before and after conducting the intervention in both study groups. The obtained data were analyzed by SPSS using descriptive statistics, Student's t-test, Paired Samples t-test, and Chi-squared test at a significance level of $\mathrm{P}<0.05$.

Findings The mean $\pm S D$ age of the investigated patients in the intervention and control groups were $37.13 \pm 31.35$ and $44.15 \pm 51.65$ years, respectively. Most frequent operation type was inguinal hernia in both study groups. Before the intervention, there was no significant difference between the two groups in terms of anxiety ( $P>0.05)$; however, after the intervention, there was a significant difference between the study groups in terms of apparent and total anxiety $(P<0.05)$. Furthermore, there was no significant difference between the study groups, in terms of latent anxiety ( $P>0.05)$.

Conclusion Aromatherapy with rose is effective in decreasing the apparent anxiety of patients before surgery. Thus, it is recommended to be used as a complementary, inexpensive, and non-invasive treatment at healthcare centers.

\section{Extended Abstract}

\section{Introduction}

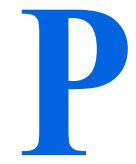

reoperative anxiety is a part of the surgical experience and a pervasive problem [1] that affects patients' health [2]. Anxiety could delay the patient's recovery due to reduced ability to fight infections, delayed wound healing, and the exacerbation of stress [3]. Furthermore, the effective dose of analgesics and anesthetics increase with anxiety [4].

Previous studies have indicated that red rose essential oil has an antidepressant effect; therefore, it could be effective in treating postpartum depression and the symptoms of Premenstrual Syndrome (PMS). Additionally, applying rose essential oil has been effective in reducing the severity of anxiety in mothers and infants without any adverse effects

\section{* Corresponding Author:}

Hossein Jeddi

Address: Student Research Committee, Gonabad University of Medical Sciences, Gonabad, Iran.

Tel: +98 (51) 57225027

E-mail: one_hossein@yahoo.com 
$[5,6]$. Mohebi-Tabar et al. also supported that this plant has anti-anxiety effects and relieves biopsychological pain [7]. Red rose essential oil has a significant impact on reducing anxiety in nulliparous women during the active phase of labor [8]. In Iranian traditional medicine, using red rose has been recommended to improve migraine pain [9], dysmenorrhea [10], musculoskeletal pain, morning sickness in pregnant women, depression, and PMS [11].

The present study aimed to investigate the effect of aromatherapy with red rose essential oil on the severity of anxiety before abdominal surgery.

\section{Materials and Methods}

This clinical trial was conducted on patients undergoing abdominal surgery who were hospitalized in 15-Khordad Hospital in Bidokht of Gonabad City, Iran, in 2017. In total, 90 patients undergoing inpatient abdominal surgery were selected by random sampling method. EmsiG oral thermometer, CF02 model; sphygmomanometer; ALPK2 stethoscope; Spielberger inventory, and red rose essential oil were implemented in the present research.

\section{Results}

The Mean \pm SD age of the investigated patients in the intervention and placebo groups was $37.13 \pm 31.35$ and $44.15 \pm 51.65$ years, respectively. The most frequent type of operation in both groups was inguinal hernia surgery. Prior to the intervention, there were no statistically significant differences between the study groups in terms of the severity of anxiety $(\mathrm{P}>0.05)$; however, after the intervention, the statistical difference was significant in terms of the severity of apparent and total anxiety scores $(\mathrm{P}<0.05)$, the same value was not significant in terms of the severity of latent anxiety $(\mathrm{P}>0.05)$ (Tables $1 \& 2)$.

Table 1. Comparing latent and apparent anxiety between the study groups before and after the intervention

\begin{tabular}{|c|c|c|c|c|c|c|c|}
\hline \multirow{3}{*}{ Anxiety } & \multirow{3}{*}{ Study Phase } & \multirow{3}{*}{ Group } & \multirow{3}{*}{ Mean士SD } & \multicolumn{2}{|c|}{ Between-groups } & \multirow{2}{*}{\multicolumn{2}{|c|}{$\frac{\text { Intergroup }}{\text { (Statistical Test, P) }}$}} \\
\hline & & & & \multirow[b]{2}{*}{ Statistical Test } & \multirow[b]{2}{*}{$\mathbf{P}$} & & \\
\hline & & & & & & $\begin{array}{c}\text { Red Rose } \\
\text { Aromatherapy } \\
\text { Group }\end{array}$ & $\begin{array}{c}\text { Placebo } \\
\text { Group }\end{array}$ \\
\hline \multirow{4}{*}{ Apparent } & \multirow{2}{*}{ Before intervention } & Red rose & $51.05 \pm 44.7$ & Independent & \multirow{2}{*}{0.079} & \multirow{4}{*}{$\begin{array}{c}\text { Paired Samples } \\
\text { t-test } \\
0.005\end{array}$} & \multirow{4}{*}{$\begin{array}{c}\text { Paired } \\
\text { Samples } \\
\text { t-test } \\
<0.001\end{array}$} \\
\hline & & Placebo & $64.56 \pm 47.7$ & Samples t-test & & & \\
\hline & \multirow{2}{*}{ After intervention } & Red rose & $64.94 \pm 42.7$ & Independent & \multirow{2}{*}{0.005} & & \\
\hline & & Placebo & $98.43 \pm 46.7$ & Samples t-test & & & \\
\hline \multirow{4}{*}{ latent } & \multirow{2}{*}{ Before intervention } & Red rose & $24.28 \pm 45.8$ & Independent & 0 & \multirow{4}{*}{$\begin{array}{c}\text { Paired Samples } \\
\text { t-test } \\
<0.001\end{array}$} & \multirow{4}{*}{$\begin{array}{c}\text { Paired } \\
\text { Samples } \\
\text { t-test } \\
0.08\end{array}$} \\
\hline & & Placebo & $64.67 \pm 48.9$ & Samples t & $0.1 / 3$ & & \\
\hline & \multirow{2}{*}{ After intervention } & Red rose & $24.32 \pm 44.8$ & Independent & \multirow{2}{*}{0.068} & & \\
\hline & & Placebo & $47.55 \pm 48.9$ & Samples t-test & & & \\
\hline & & & & & & $\begin{array}{l}\text { uarterly of } \\
\text { he Horizon of } \mathrm{N}\end{array}$ & cal Scie \\
\hline
\end{tabular}

Table 2. Comparing the vital signs of patients in the study groups before and after the intervention

\begin{tabular}{|c|c|c|c|c|c|c|}
\hline \multirow{3}{*}{$\begin{array}{c}\text { Group } \\
\text { Study Phase } \\
\text { Vital Signs }\end{array}$} & \multicolumn{3}{|c|}{ Red Rose Aromatherapy } & \multirow{2}{*}{\multicolumn{3}{|c|}{ Placebo }} \\
\hline & \multicolumn{2}{|c|}{ Mean $\pm S D$} & \multirow[b]{2}{*}{$\begin{array}{l}\text { Paired Samples } \\
\text { t-test, P }\end{array}$} & & & \\
\hline & Pre-test & Post-test & & $\begin{array}{c}\text { Before } \\
\text { Intervention }\end{array}$ & $\begin{array}{c}\text { After } \\
\text { Intervention }\end{array}$ & $\begin{array}{l}\text { Paired Samples } \\
\text { t-test, P }\end{array}$ \\
\hline Pulse & $8.035 \pm 77.36$ & $6.048 \pm 76.87$ & 0.027 & $7.105 \pm 78.49$ & $7.248 \pm 78.47$ & 1.000 \\
\hline Breathing & $1.581 \pm 15.00$ & $1.588 \pm 14.98$ & 0.317 & $2.199 \pm 15.73$ & $2.170 \pm 15.71$ & 0.310 \\
\hline $\begin{array}{l}\text { Systolic blood } \\
\text { pressure }\end{array}$ & $12.17 \pm 128.66$ & $12.10 \pm 128.12$ & 0.083 & $11.36 \pm 127.55$ & $11.41 \pm 127.44$ & 0.561 \\
\hline $\begin{array}{l}\text { Diastolic blood } \\
\text { pressure }\end{array}$ & $65.6 \pm 66.76$ & $6.60 \pm 76.22$ & 0.317 & $5.75 \pm 76.22$ & $5.75 \pm 76.22$ & 1.000 \\
\hline
\end{tabular}




\section{Discussion}

The present study results suggested that red rose essential reduced the severity of apparent anxiety before surgery, i.e. consistent with other studies $[5,8,12]$. Moreover, the levels of apparent and latent anxiety in patients were moderate to high before the intervention. A study explored the effect of inhaled aromatherapy on the severity of patients' anxiety before surgery. Accordingly, the mean severity of apparent anxiety before surgery was moderate to high $(51.00 \pm 8.94)$ [13]. In Kohnegi's study, however, the mean score of apparent anxiety was relatively high. This discrepancy may be due to differences in the surgery type performed on patients undergoing coronary artery bypass graft surgery; it naturally results in more severe anxiety, compared to abdominal surgery [14].

It is recommended that studies be performed with a larger sample size; the effect of rose essential oil on other anxietygenerating interventions be considered, and other psychological variables, like stress, be assessed.

\section{Conclusion}

Factors, such as the intervention type, the therapeutic outcomes, patient's characteristics, family support, and insurance coverage also affect the severity of the patient's anxiety. These elements could impact the research results, i.e. beyond the researchers' control. Another study limitation was the impossibility of blinding the specimens and researchers, as well as the highly stressful preoperative condition, which may have complicated the studied patients' assessment of their anxiety severity.

\section{Ethical Considerations}

\section{Compliance with ethical guidelines}

This project was approved by the Ethics Committee of the Regional Ethics Committee of Gonabad University of Medical Sciences (Code: IR.GMU.REC.1395.43). Moreover, all the instructions approved by this committee have been considered in this study.

\section{Funding}

The present research was funded by the Student Research Committee of Gonabad University of Medical Sciences.

\section{Authors' contributions}

Conceptualization: Samaneh Najafi, Moosa Sajjadi, Amirreza Nasirzadeh, Hossein Jeddi; Methodology, Sa- maneh Najafi, Moosa Sajadi; Investigation: Hossein Jeddi, Writing-original draft: Samaneh Najafi, Moosa Sajjadi, Amirreza Nasirzadeh, Hossein Jeddi; Writing-review \& editing: Samaneh Najafi, Moosa Sajjadi, Amirreza Nasirzadeh, Hossein Jeddi; Funding acquisition: Samaneh Najafi, Moosa Sajadi; Supervision: Samaneh Najafi, Moosa Sajadi.

\section{Conflicts of interest}

The authors declared no conflicts of interest

\section{Acknowledgements}

We express our gratitude for the support of the ViceChancellor for Research and Technology, as well as the Student Research Committee of Gonabad University of Medical Sciences and the esteemed staff of the surgical wards of Gonabad 15-Khordad Hospital and the patients who contributed to this research. 


\title{
تأثير رايحهدرمانى استنشاقى با كَل سرخ بر شدت اضطراب قبل از عمل جراحى شكم: يكى كارآزمايع بالينى شاهددار تصادفى استشى
}

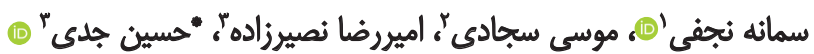

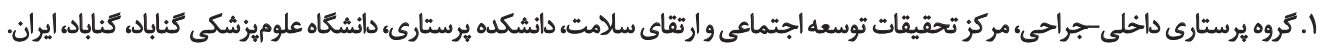

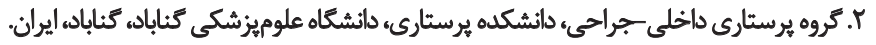

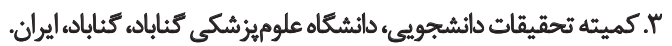

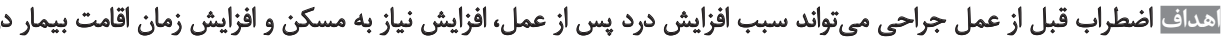

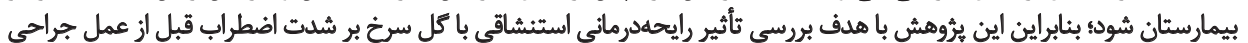
شكم انجام شد.

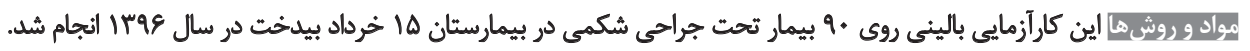

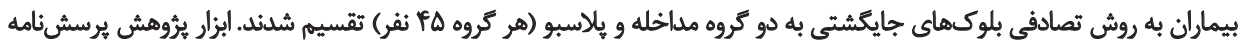

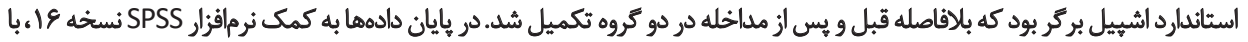

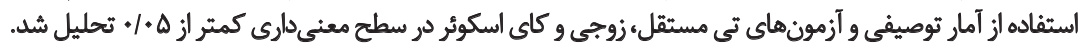

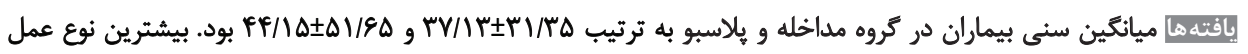

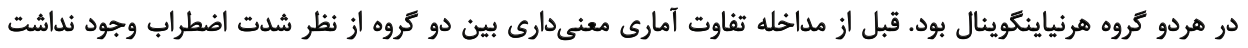

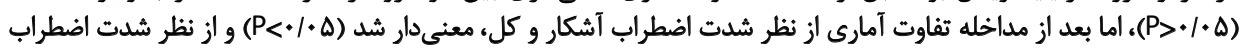

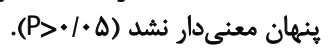

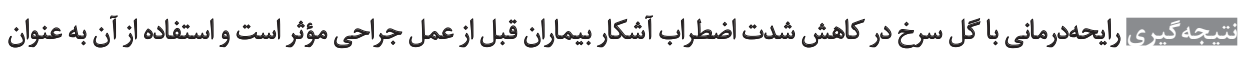

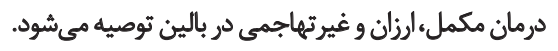

تاريخ دريافت: و ارديبهشت

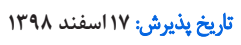

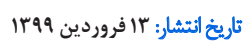

كليدوازوها: كل سرخ، رايحهدرمانى الئي

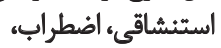
عمل جراحي

افزايش مىيابد. براى مقابله با اين موضوع از داروهاى كاهنده

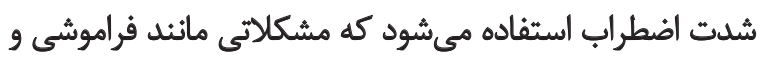

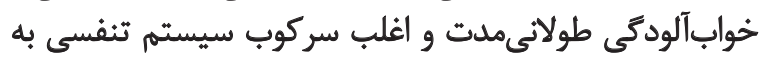

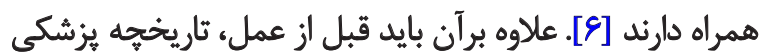

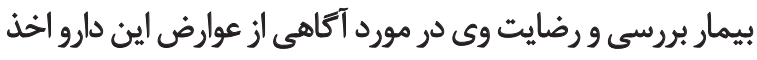

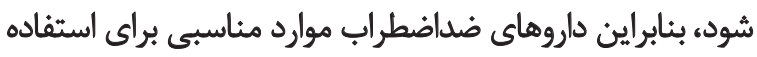

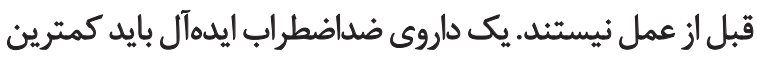

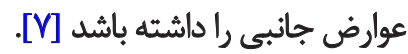

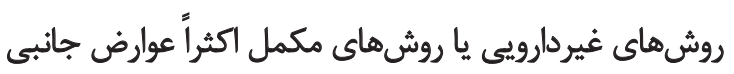

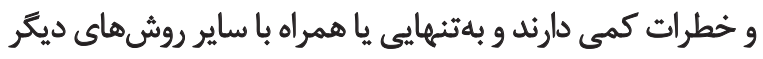

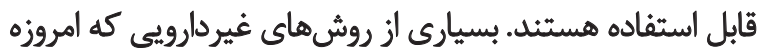

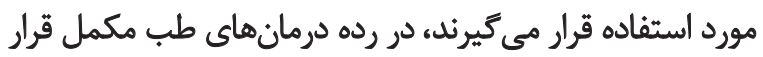

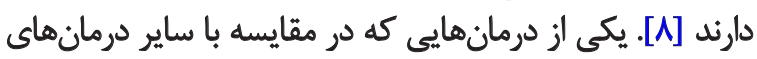

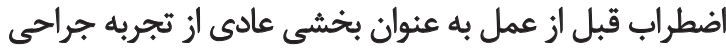

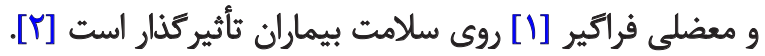

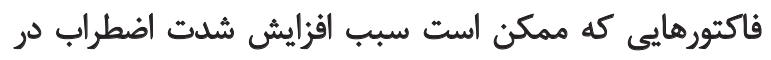

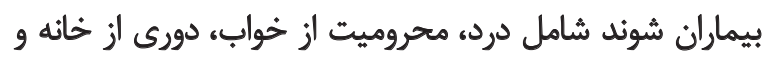

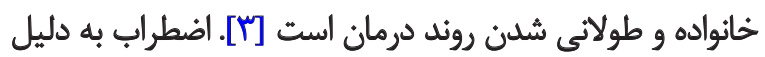

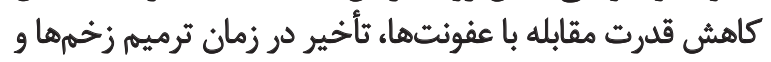

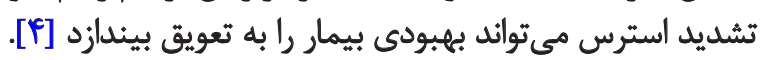

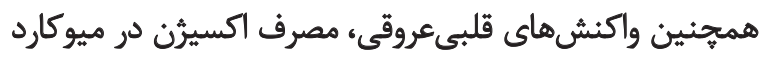

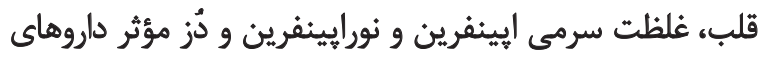

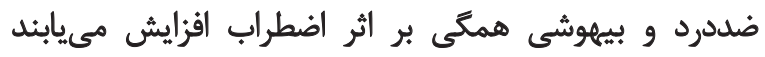

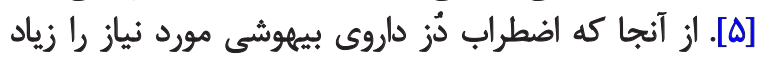

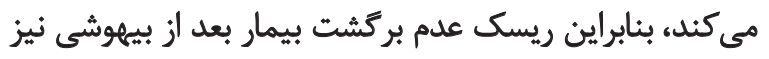


توجهي دارد [19 ]. در طب سنتى ايرانى نيز استفاده از كل سرخ

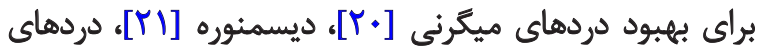

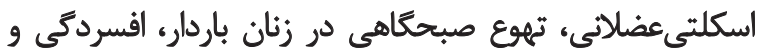

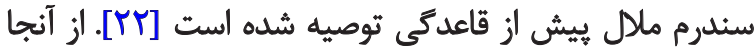

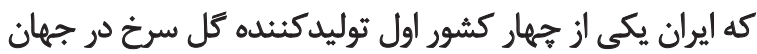

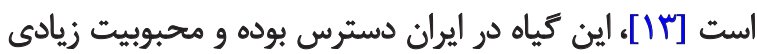

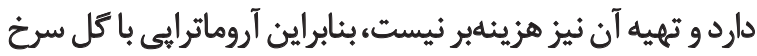

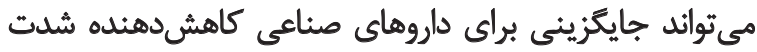

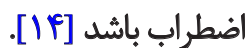

با توجه به اهميث كاهش شدت اضطراب در بيماران قبل از

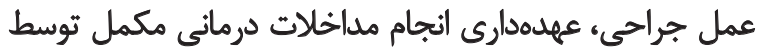

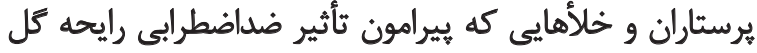

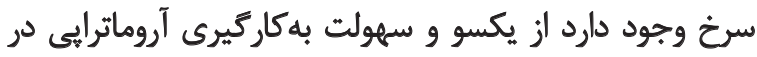

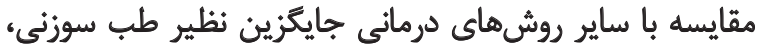

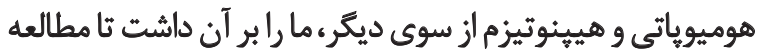

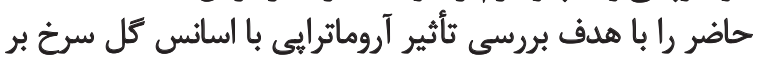

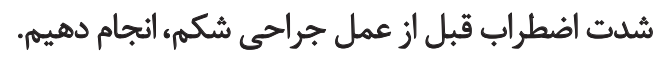

\section{مواد وروشها}

اين كارآزمايى بالينى شاهدار تصادفى روى •9 بيمار تحت

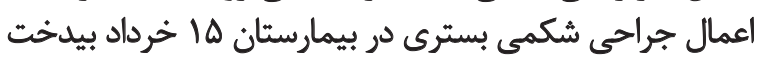

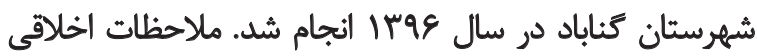

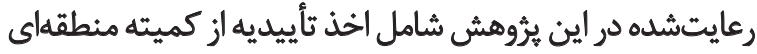

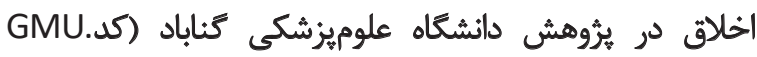

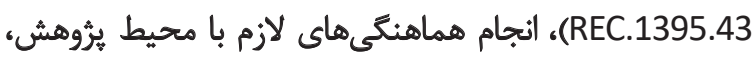

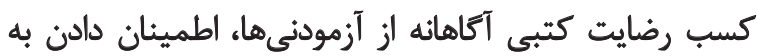

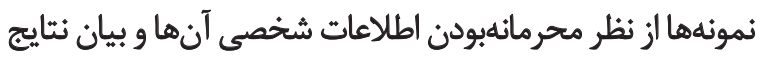

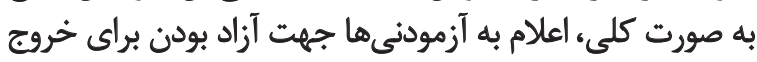

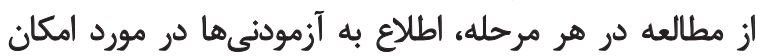

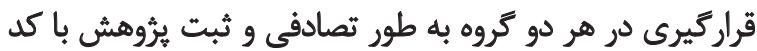

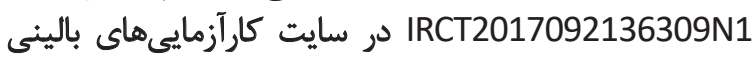

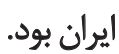

بيماران با استفاده از روش نمونهكيرى دردسترس انتخاب

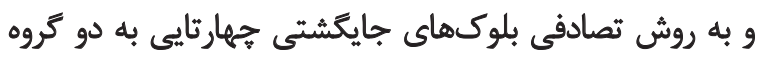

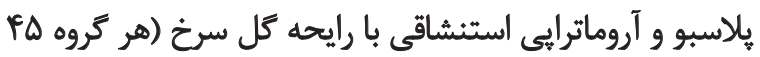

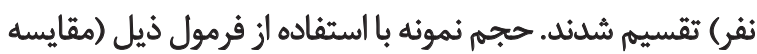

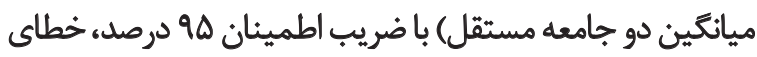

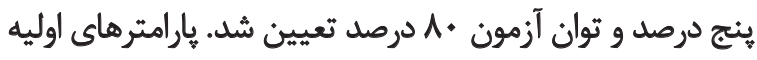

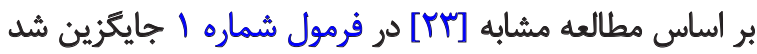

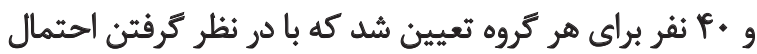

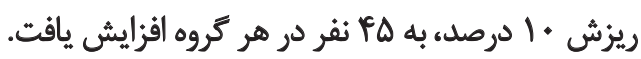

$$
\text { 1. } n=\frac{\left(Z_{1 \frac{a}{2}}+Z_{1-\beta}\right)^{2}\left(S^{2}{ }_{1}+S^{2}\right)}{\left(\mu_{1}-\mu_{2}\right)^{2}}=\frac{7.84 \times\left(6.1^{2}+5.5^{2}\right)}{(22.5-18.85)^{2}} \cong 40
$$

طب مكمل در رابطه با شدت اضطراب در سال هاى اخير در اكثر

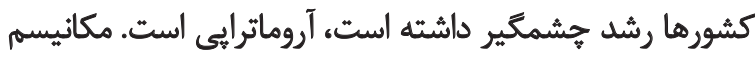

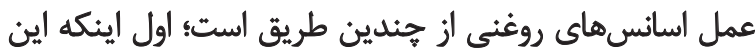

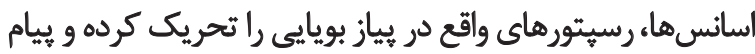

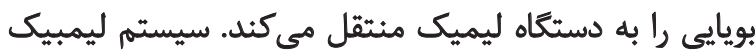

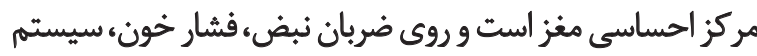
تنفسى و هاسخ به استرس مؤثر است [9"]

مكانيسم ديكر رايحهدمانى از طريق جذب يوستى است.

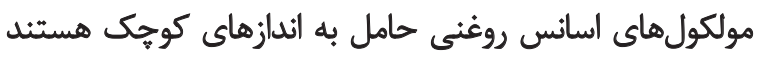

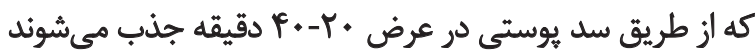

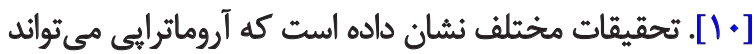

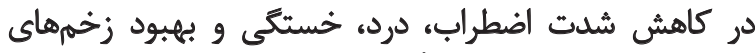

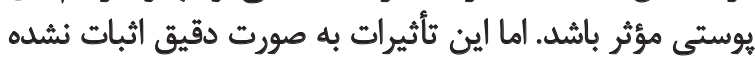

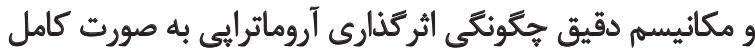

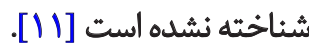

مقبوليت بيشتر و عوارض جانبى كمتر روشهاى غيردارئ دارويى

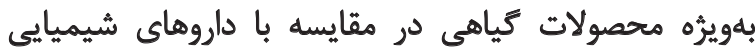

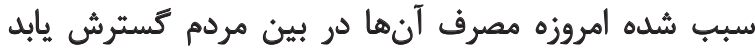

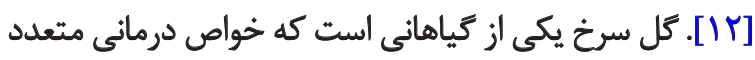

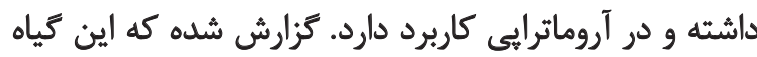

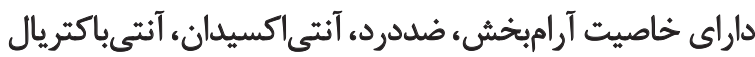

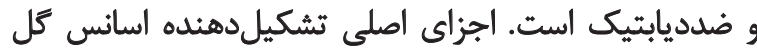

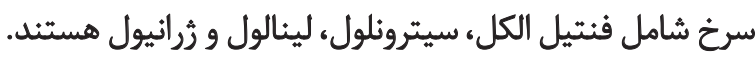

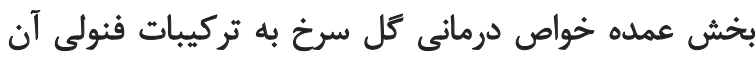

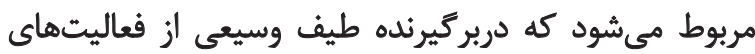

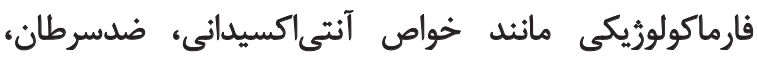

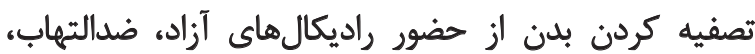

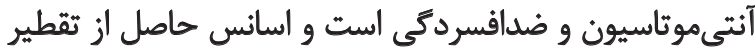

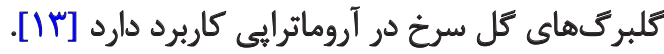

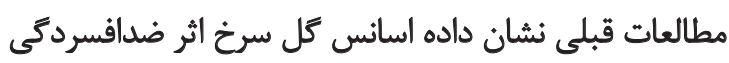

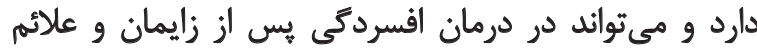

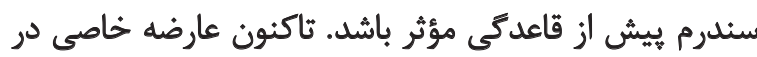

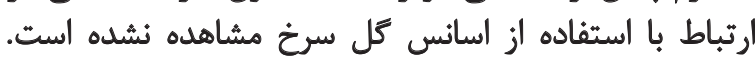

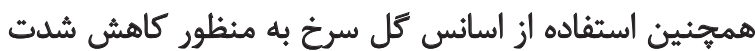

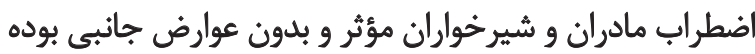

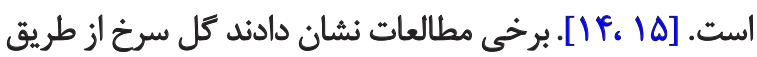

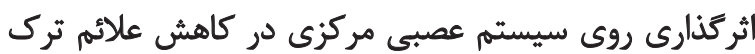

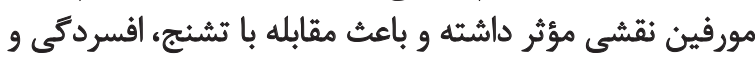

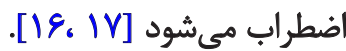

نتايج بررسى هاى انجامشده توسط محبيىتبار و همكاران نيز

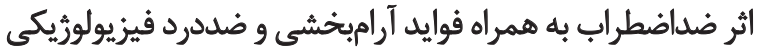

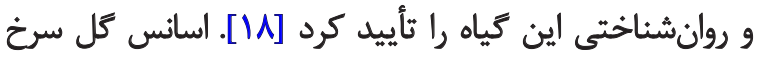

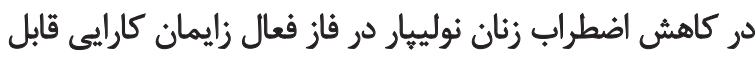


بيانكَّ افزايش نمره اضطراب است؛ بدين صورت كه كسب نمره

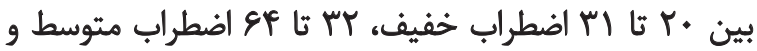

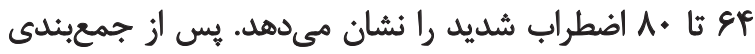

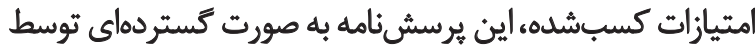
روانشناسان، متخصصان و محققان استفاده شده است و اعتبار

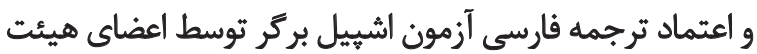

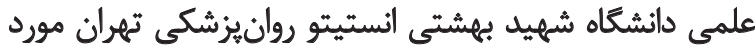

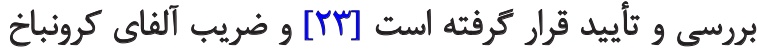

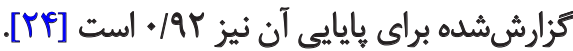
يروهشكران يس از كسب اجازه از مسئولين ذئربط و كرفتن

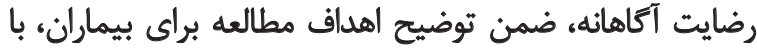

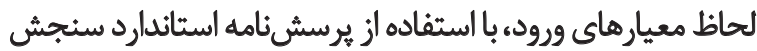

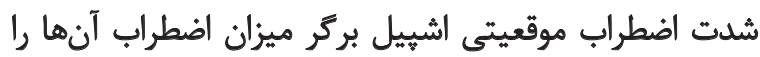

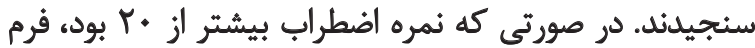

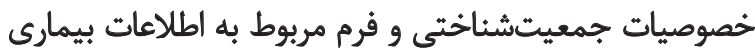

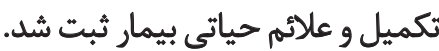

با توجه به اينكه بيمار در كدام تروه قرار مئرترفت، مداخله

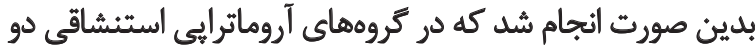

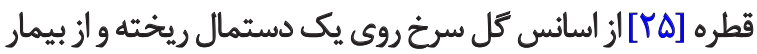

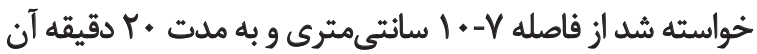

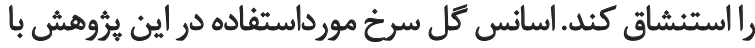

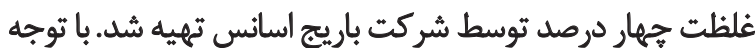

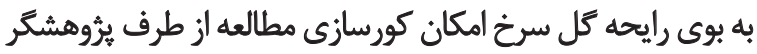

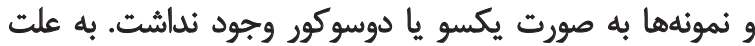

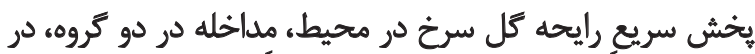

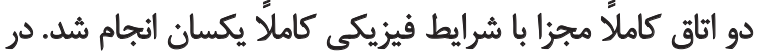

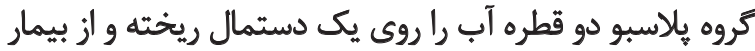

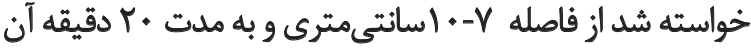

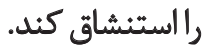

دليل انتخاب آب به عنوان يلاسبو نداشتن بو، عطر و رايحه

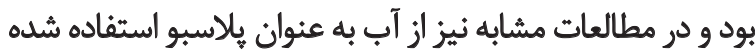

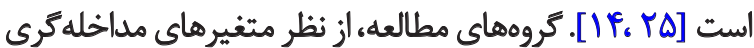

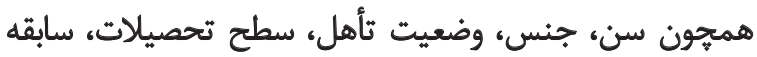

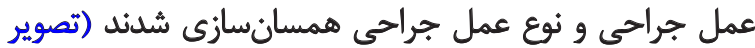

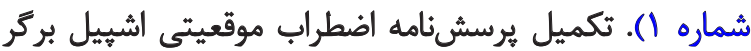

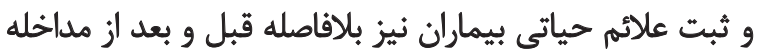

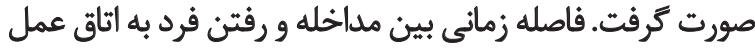

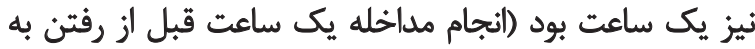

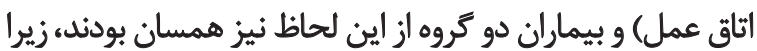

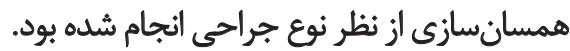

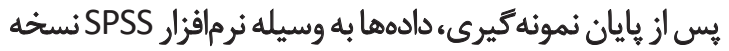

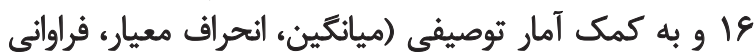
مطلق و نسبى) و آزمونهاي آمار تحليلى نظير تى مسينى مستقل و

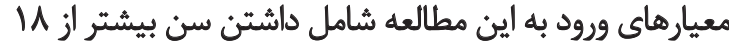

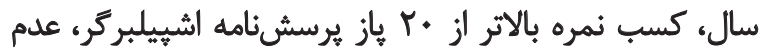

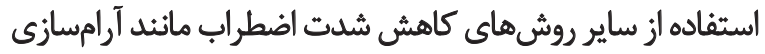

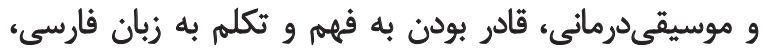

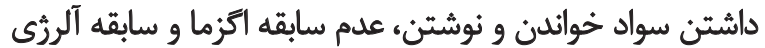

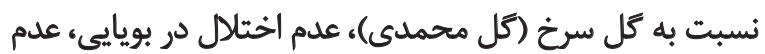

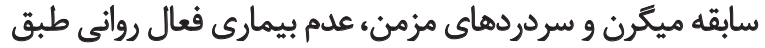

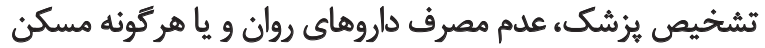

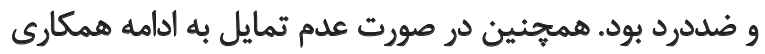

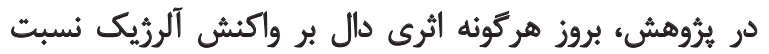

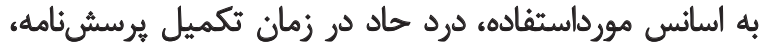

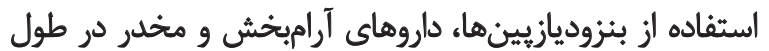
مداخله، بيماران از مطالعه خارج مي إنداند.

كردآورى دادهها از طريق مصاحبه (در مواردى كه مددجويان

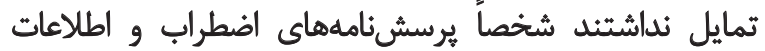

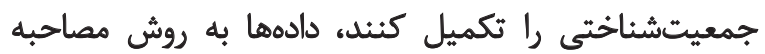

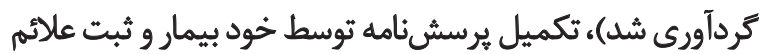

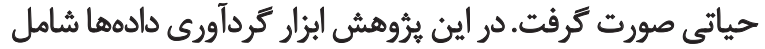

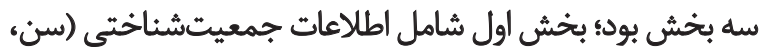

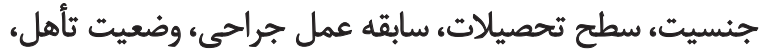

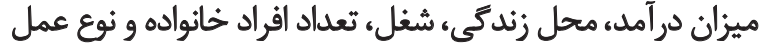

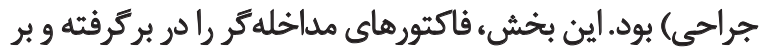

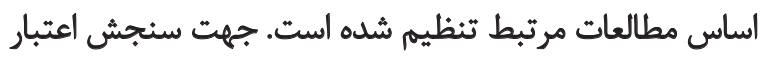

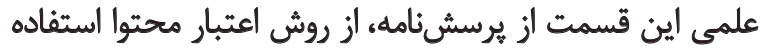

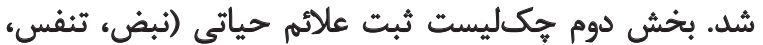
درجه حرارت و فشار خون) بودي

براى اندازميرى درجه حرارت و فشار خون به ترتيب از

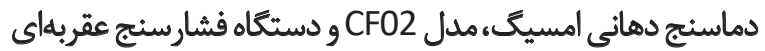
و كوشى يزشكى مدل ALPK2 ساخت كشور راين برائ تمام

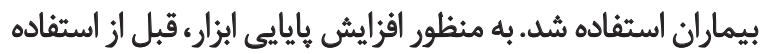

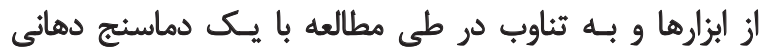

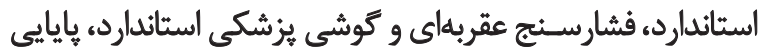

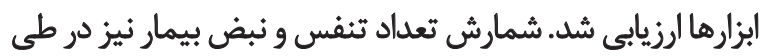

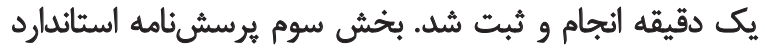

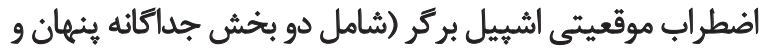

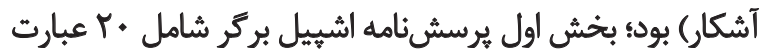

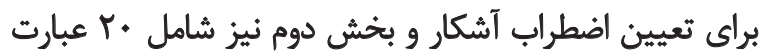

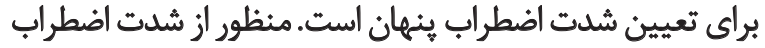

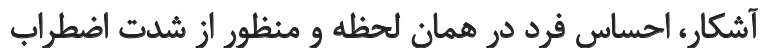

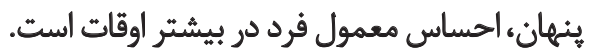

سؤالات بر اساس مقياس ليكرت جهاردر جهاى از يك تا جهار

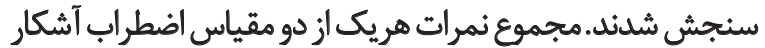

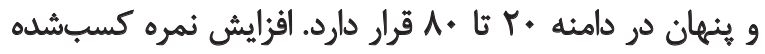


كل و آشكار در هر دو گروه قبل و بعد از مداخله اختلاف معنادار

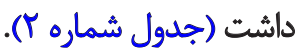

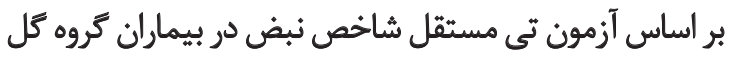

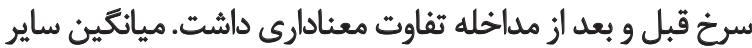

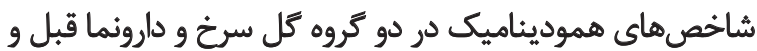

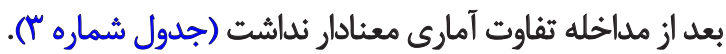
بر اساس نتايج ارتباط آمارى معنى دارى بين جنس، سن،

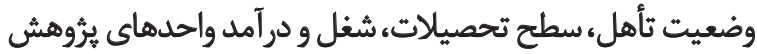

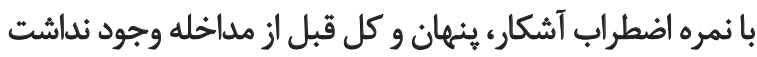

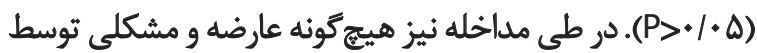

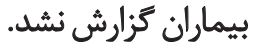

بحث

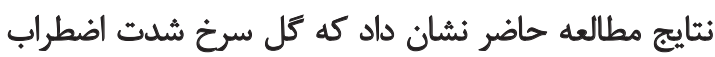

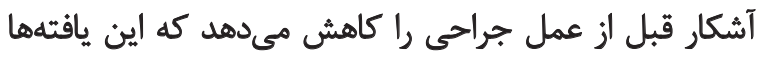

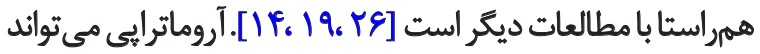

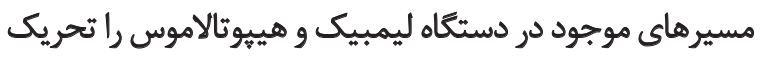

زوجى و كاى اسكوئر تجزيه و تحليل شد. سطح معنادارى كمتر

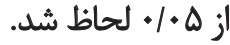

يافتهها

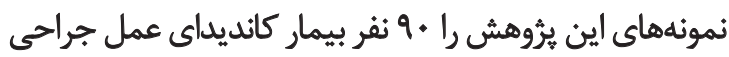

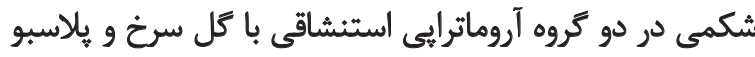

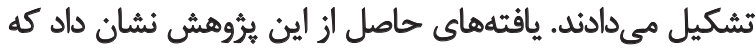

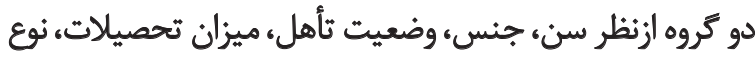

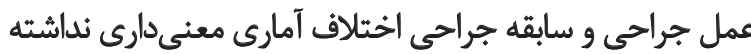
(P> / / A) و همسان هستند (جدول شماره ()). آزمون تى مستقل نشان داد كه ميانگين نمره اضطراب كل،

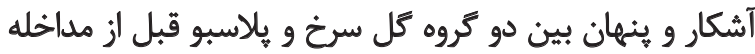

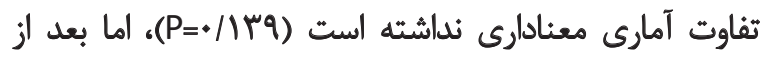

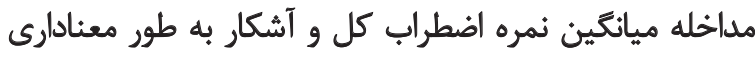

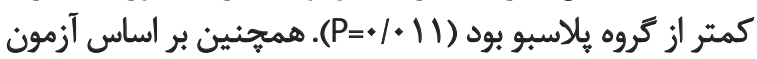

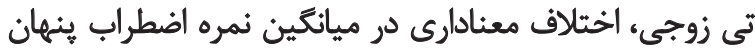

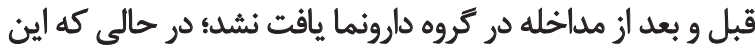

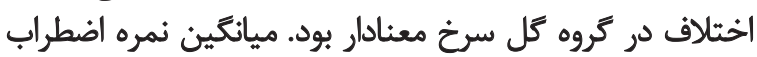

جدول ا. مقايسه اطلاعات جمعيتشناختى واحدهاي يثروهش در دو گروه موردمطالعه

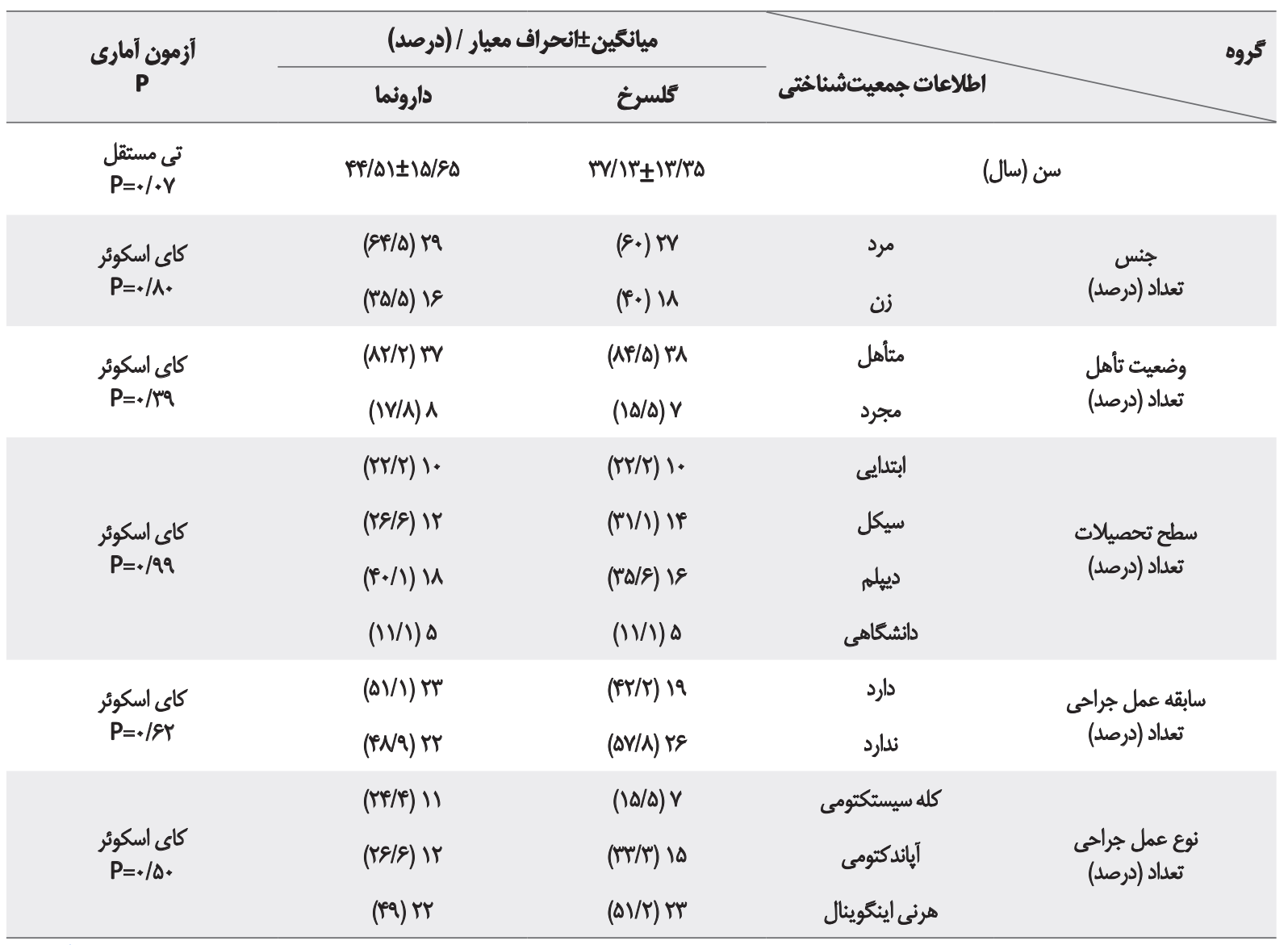


جدول r. مقايسه اضطراب ينهان و آشكار در دو گروه قبل و بعد از مداخله

\begin{tabular}{|c|c|c|c|c|c|c|c|}
\hline \multicolumn{2}{|c|}{ دورن كروهى } & \multicolumn{2}{|c|}{ بين كَروهى } & \multirow{3}{*}{ (ميانكين } & \multirow{3}{*}{$\operatorname{los}^{\infty}$} & \multirow{3}{*}{ فاز مطالعه } & \multirow{3}{*}{ اضطراب } \\
\hline \multicolumn{2}{|c|}{ أزمون آمارى (P) } & $D$ & & & & & \\
\hline كيوه هاسيو & كَّوه كل سنْ & & & & & & \\
\hline \multirow{7}{*}{ تى زوجى } & & & & $\Delta V / \cdot \Delta \pm F F / V$ & كل سرخ & & \\
\hline & & $\mathrm{P}=* 1+\mathrm{Vq}$ & تى مستثل & & & قبل از مداخله & \\
\hline & & & & $F V / V \pm g Y / \Delta F$ & يالاسبو & & \\
\hline & 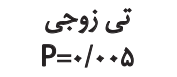 & & & & & & آشكار \\
\hline & & & & $g e / q T \pm T / N$ & كَل سن & & \\
\hline & & $\mathrm{P}=\star / * \Delta$ & تى مستثل & & & بعد ازز مداخله & \\
\hline & & & & Whew \pm fe/V & يالاسبو & & \\
\hline \multirow{7}{*}{$\begin{array}{l}\text { تى زوجى } \\
\text { P=॰1•1 }\end{array}$} & & & & re/rAtFa/A & كَل سغ & & \\
\hline & & $\mathrm{P}=. / 1 V \Delta$ & تي مستقل & & & قبل از مداخله & \\
\hline & & & & gFientFa & هياسبو & & \\
\hline & $\begin{array}{l}\text { تى زوجى } \\
P<+1 .+1\end{array}$ & & & & & & ينهان \\
\hline & & & & $r E / M T \pm F \varphi / A$ & كل سخغ & & \\
\hline & & $\mathrm{P}=.1 .81$ & تي مستقل & & & بعد از مداخله & \\
\hline & & & & $P V / \Delta \Delta \pm \Psi N q$ & بالاسبو & & \\
\hline
\end{tabular}

جدول ب. مقايسه علاثم حياتئ بيماران در دو كروه قبل و بعد از مداخله

\begin{tabular}{|c|c|c|c|c|c|c|}
\hline \multicolumn{3}{|c|}{ إرونما } & \multicolumn{3}{|c|}{ كَّل سيخ } & \multirow{2}{*}{ عائم حيازتى } \\
\hline P أزمون تي زوجى & بعد از مداخله & قبل از مداخله & أزمون تى زوجى & بعد از مداخله & قبل از مداخله & \\
\hline $\mathrm{P}=1 / \ldots$ & YNTEEV/YEA & $V N=q \pm V / N \cdot \Delta$ & $\mathrm{P}=* / . \mathrm{TV}$ & VelAVI\&/. IA & Whetel.ro & نبض \\
\hline $\mathrm{P}=\cdot\left({ }^{\prime}\right)$ & $\mid Q / Y \backslash \pm Y / I V$ & $10 / N+ \pm T / 199$ & $\mathrm{P}=. /$ IIV & $1 F / U \pm 1 / \Delta M$ & $|Q / \cdot . \pm| / \Delta A \mid$ & تنفس \\
\hline $\mathrm{P}=\cdot|\Delta\rangle \mid$ & $|r V / F= \pm| 1 / F \mid$ & $\mid r W / \Delta \Delta \pm \| / T E$ & $P=* / \cdot A r$ & IYNIYEIYM. & IYNFE IIYAV & فشارخون سيستوليك \\
\hline$P=1 / \ldots$ & $V E / T Y \pm \Delta / V \Delta$ & $V E / M Y \pm \Delta / V \Delta$ & $P=* / M I V$ & $V \varepsilon / r Y \pm g / g$. & VF/F9 $\$ 9 / 80$ & فشارخون دياستوليك \\
\hline
\end{tabular}
أنُورانم

ضداضطرابى، اثرات آرامبخشى نيز دارد [N1/]. دو تركيب اصلى

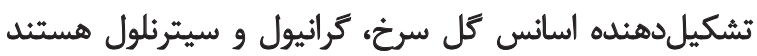

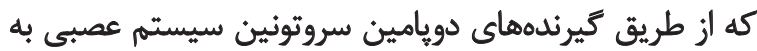

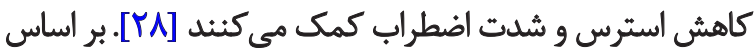

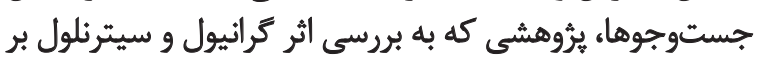

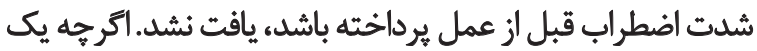

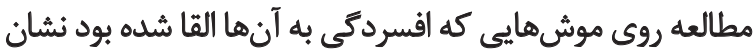

كند و منجر به كاهش آزادسازى هورمون كورثيكوتيرويين شود.

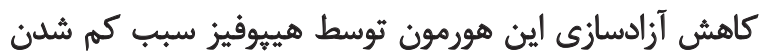

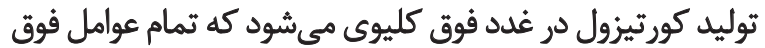

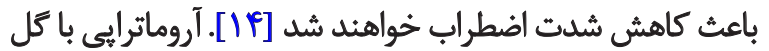

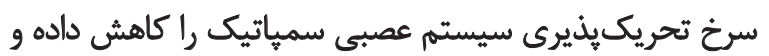

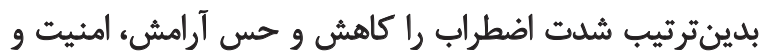

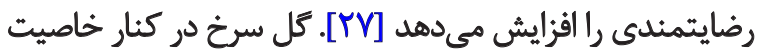




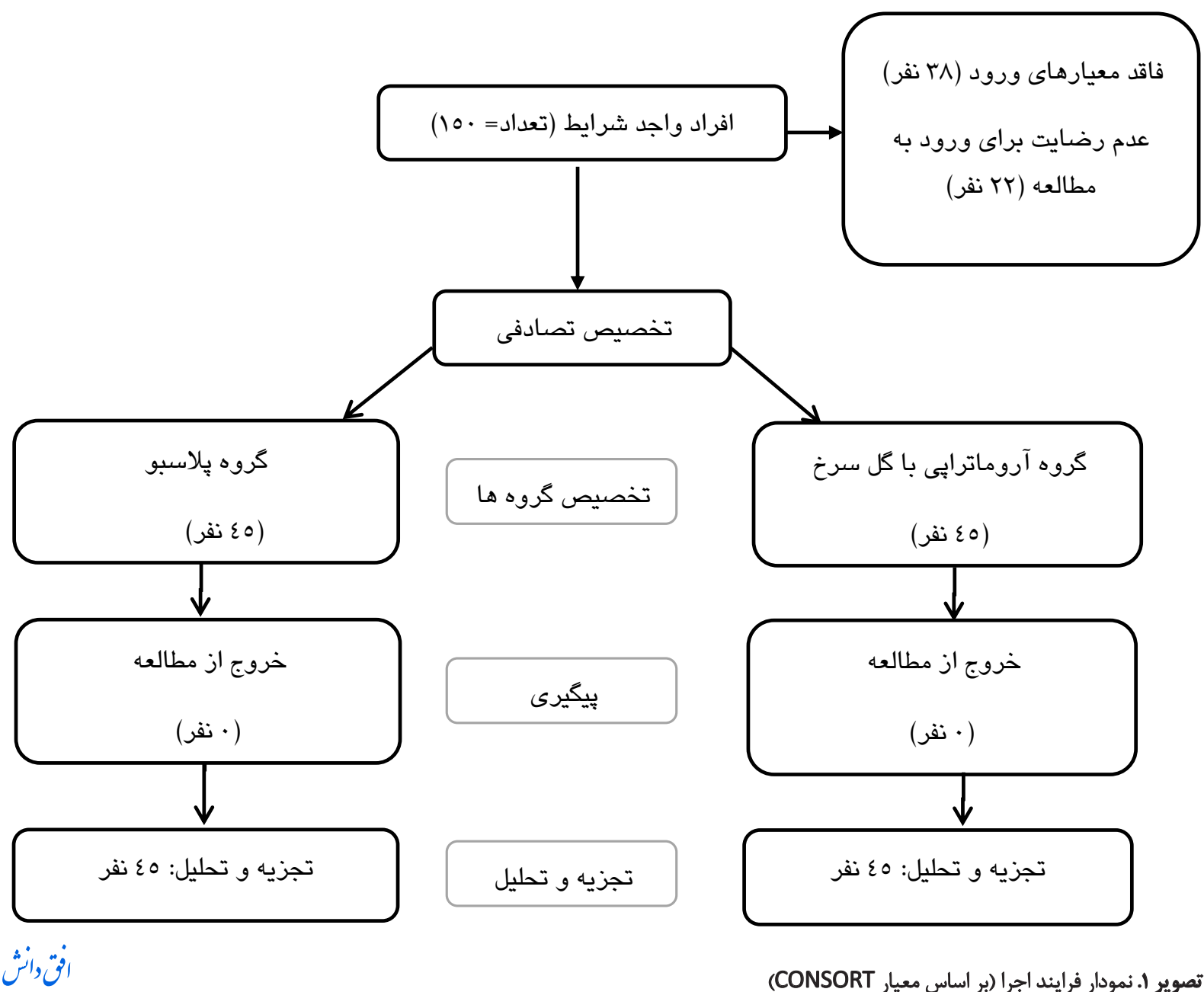

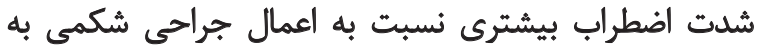

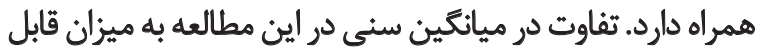

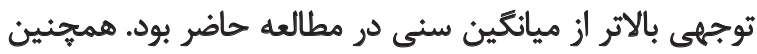

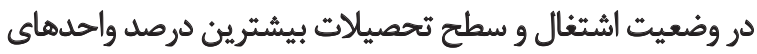

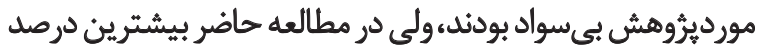

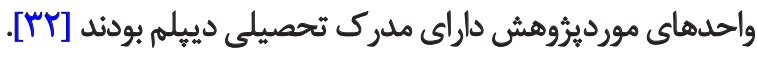
در ارتباط با تعيين شدت اضطراب، بلافاصله هي از مداخله

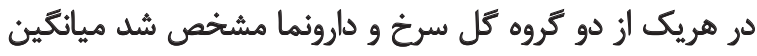

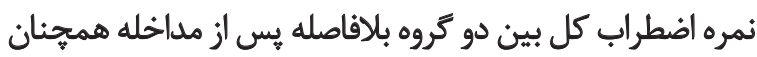

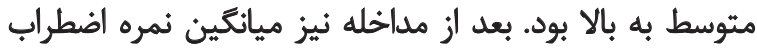

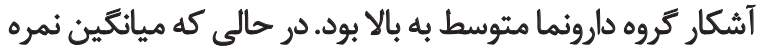

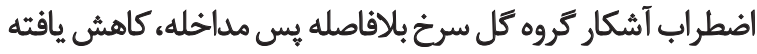

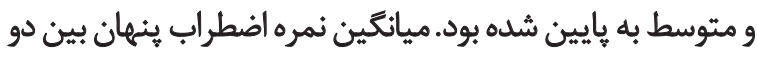

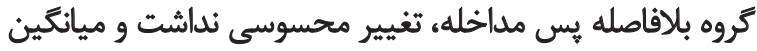

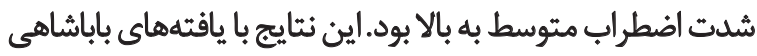

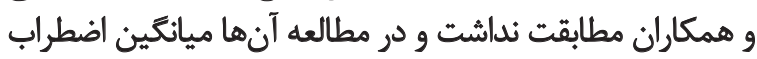

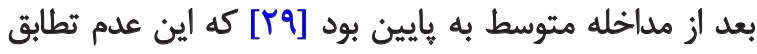

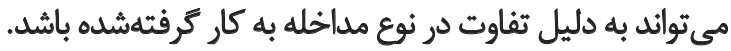

داد كل سرخ با افرزايش آنتى|كسيدانها وكاهش ليهيد يروكسيداز

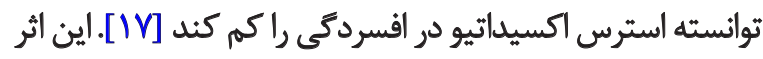

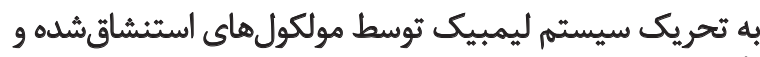

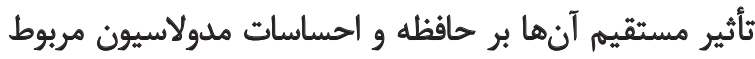

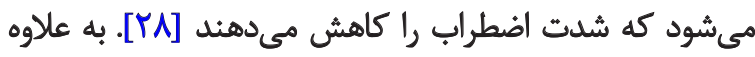

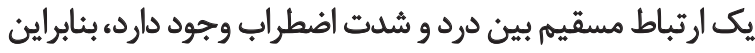

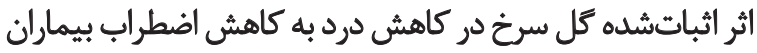

منجر خواهد شد [1f]

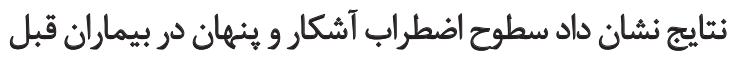

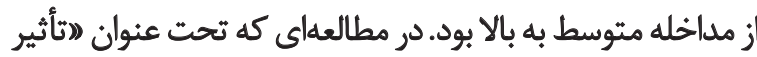

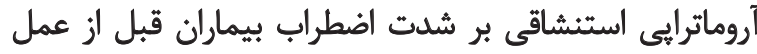

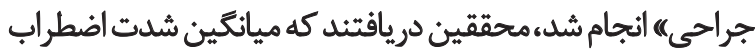

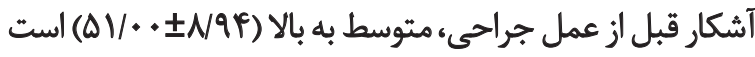

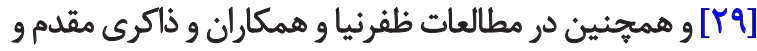

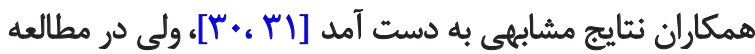

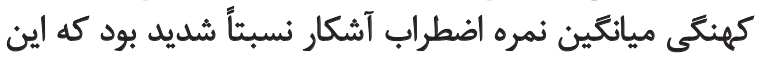

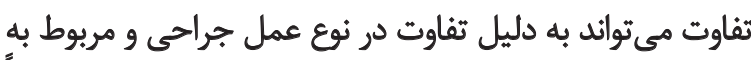

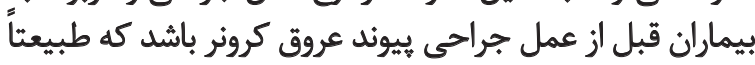




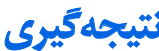

درمجموع اين مطالعه نشان داد رايحهدرمانى استنشاقى

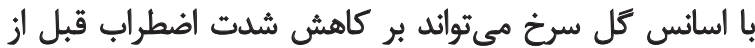

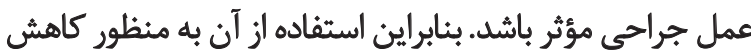

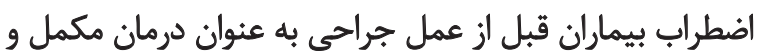

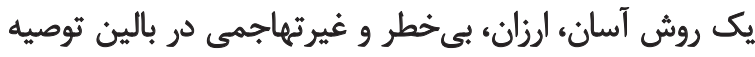

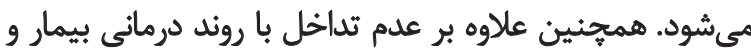

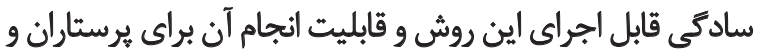

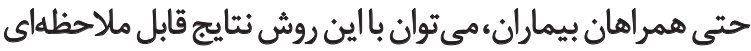

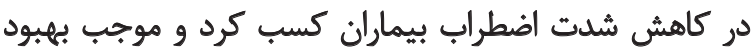

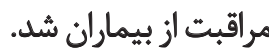

از محدوديثهاى اين يُوهش مىتوان به ارتباط بين بوها و و بوائ

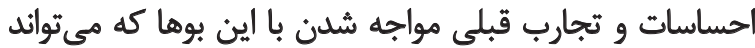

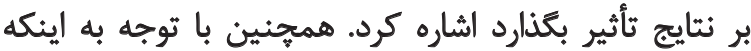

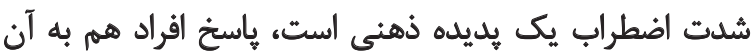

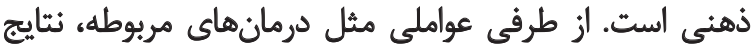

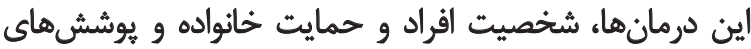

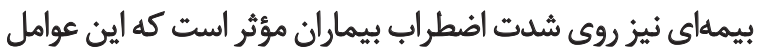

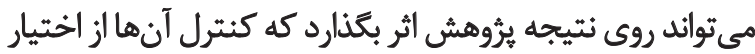

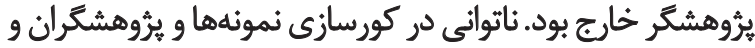

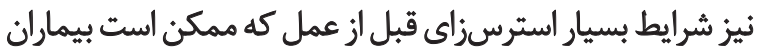

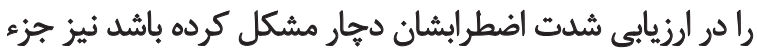

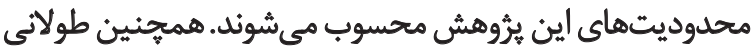

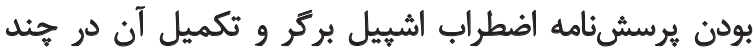

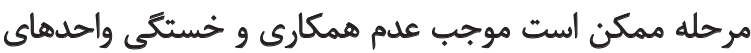

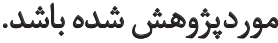

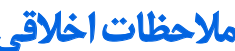 \\ يبيروى أز اصول الخالاق يُوهش}

IR.GMU. طرح داراى كد كميته اخلاق به شماره

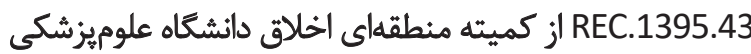

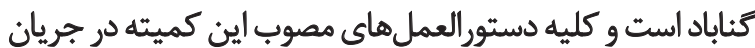

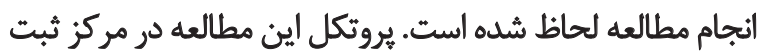

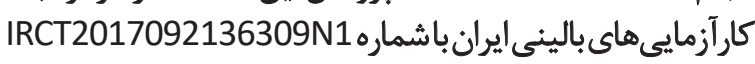
به ثُبتث روسيله أسيت.

$$
\text { ماني مالى }
$$

اين يُروهش با حمايت مالى كميته ثحقيقات دانشجويى

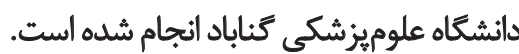

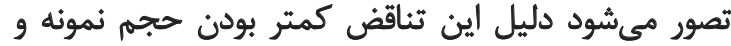

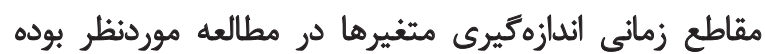

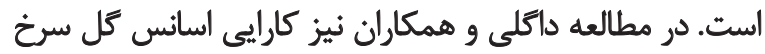

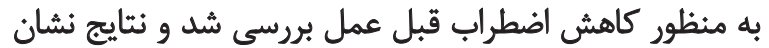

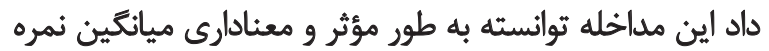

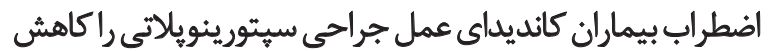

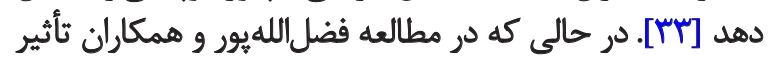

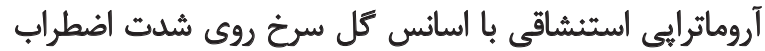

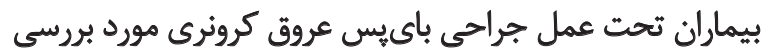

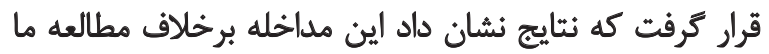

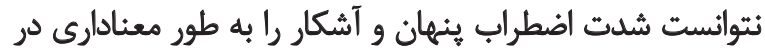

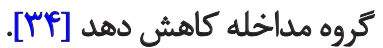
در ارتباط با مقايسه علاثم حياتى قبل و بعد از مداخله در هريك از دو كروه كل سخخ و دارونما نتايج مطالعه نشان دان داد ميان ميانكين

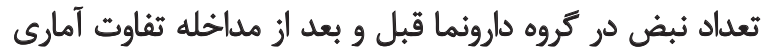

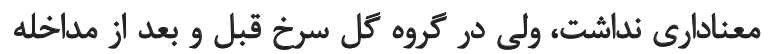
تفاوت آمارى معنادارى داشت، بنابرائن

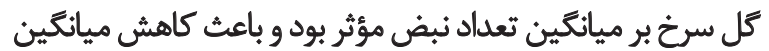

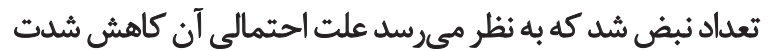

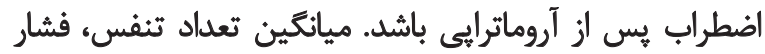

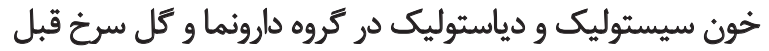

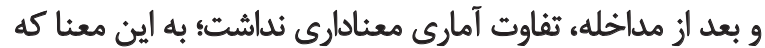

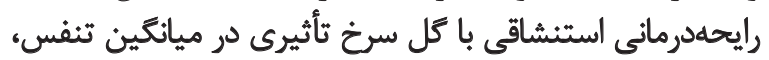

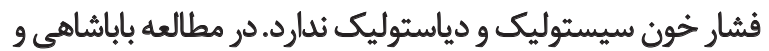

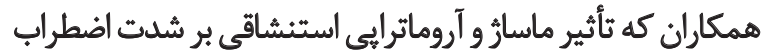

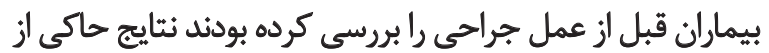

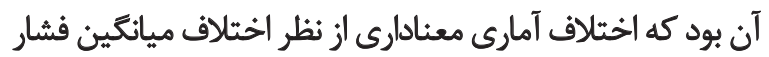

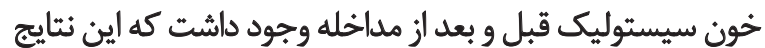
با يافتههاي ما همراستا نيست كه دليل آن ممكن است استفادئ إنداز

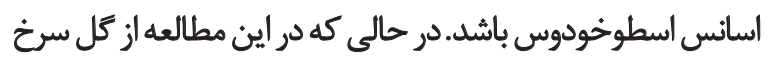

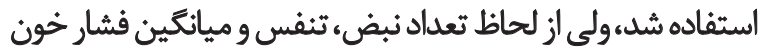

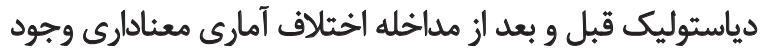
نداشت كه با نتايج مطالعه حاضر همخ وخوانى دارد. تعيين ارتباط شدت اضطراب با مشخصات جمعيتشناختى مئي

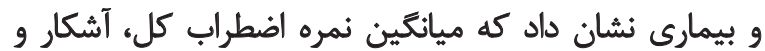

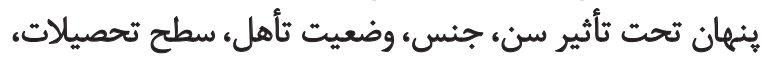

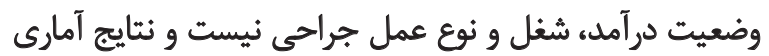

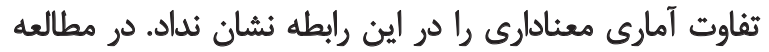

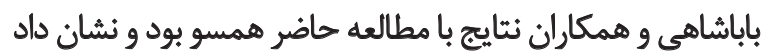

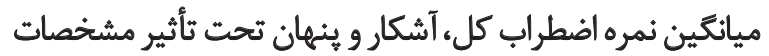

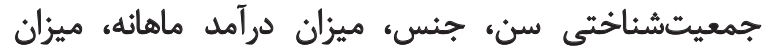

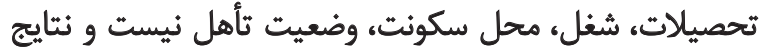

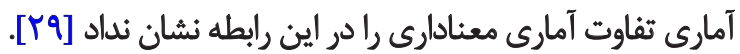




\section{مشار كت نويسئد تحان}

مفهومسازى: سمائه نجفى، موسى سجادى، اميررضا نصيرزاده،

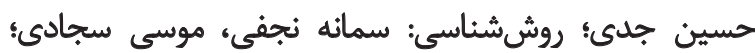

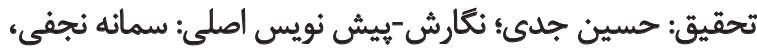

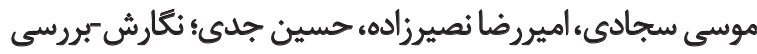

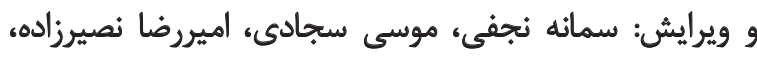

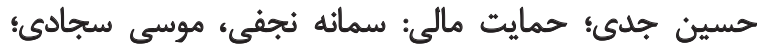

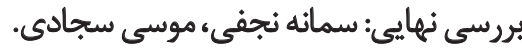

$$
\text { تمارض منافح }
$$

بنابر اظهار نويسندكان اين مقاله تعارض منافع ندارد.

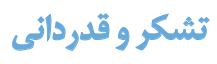

بدينوسيله از حمايت معاونت تحقيقات و فناورى و نيز

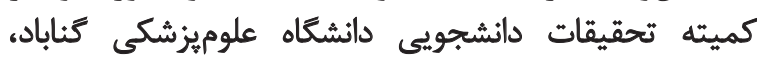

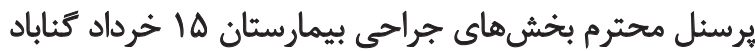

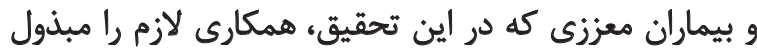
داشتند، كمال تشكر و سياس رادين داريه. 


\section{References}

[1] Burg MM, Benedetto MC, Rosenberg R, Soufer R. Presurgical depression predicts medical morbidity 6 months after coronary artery bypass graft surgery. Psychosomatic Medicine. 2003; 65(1):111-8. [DOI:10.1097/01. PSY.0000038940.33335.09] [PMID]

[2] Bailey L. Strategies for decreasing patient anxiety in the perioperative setting. AORN Journal. 2010; 92(4):445-60. [DOI:10.1016/j. aorn.2010.04.017] [PMID]

[3] Seifi Z, Beikmoradi A, Oshvandi K, Poorolajal J, Araghchian M, Safiaryan $R$. The effect of lavender essential oil on anxiety level in patients undergoing coronary artery bypass graft surgery: A double-blinded randomized clinical trial. Iranian Journal of Nursing and Midwifery Research. 2014; 19(6):574-80. [PMID] [PMCID]

[4] Rahmani Bilondi R, Najafi S, Banafsheh E, Abdolazimi Z, Tavafi M, Rahmani MR. [The effect of relaxation training using short message service on pregnant women's anxiety (Persian)]. Iranian Journal of Nursing Research. 2019; 14(1):42-9. [DOI:10.21859/ijnr-140107]

[5] Biddiss E, Knibbe TJ, McPherson A. The effectiveness of interventions aimed at reducing anxiety in health care waiting spaces: A systematic review of randomized and nonrandomized trials. Anesthesia \& Analgesia. 2014; 119(2):433-48. [DOI:10.1213/ANE.0000000000000294] [PMID]

[6] Patel SB, Kress JP. Sedation and analgesia in the mechanically ventilated patient. American Journal of Respiratory and Critical Care Medicine. 2012; 185(5):486-97. [DOI:10.1164/rccm.201102-0273CI] [PMID]

[7] Franco L, Blanck TJ, Dugan K, Kline R, Shanmugam G, Galotti A, et al. Both lavender fleur oil and unscented oil aromatherapy reduce preoperative anxiety in breast surgery patients: A randomized trial. Journal of Clinical Anesthesia. 2016; 33:243-9. [DOI:10.1016/j.jclinane.2016.02.032] [PMID]

[8] Bettiol A, Lombardi N, Marconi E, Crescioli G, Bonaiuti R, Maggini V, et al. The use of complementary and alternative medicines during breastfeeding: Results from the Herbal supplements in Breastfeeding InvesTigation (HaBIT) study. British Journal of Clinical Pharmacology. 2018; 84(9):2040-7. [DOI:10.1111/bcp.13639] [PMID] [PMCID]

[9] Abdelhakim AM, Hussein AS, Doheim MF, Sayed AK. The Effect of inhalation aromatherapy in patients undergoing cardiac surgery: A systematic review and meta-analysis of randomized controlled trials. Complementary Therapies in Medicine. 2019; 48(12):e102256. [DOI:10.1016/j. ctim.2019.102256]

[10] Micozzi MS. Fundamentals of complementary, alternative, and integrative medicine. 6th ed. St. Louis: Elsevier Health Sciences; 2018. https://books.google.com/books?id=siRyDwAAQBAJ\&dq

[11] Kyle G. Evaluating the effectiveness of aromatherapy in reducing levels of anxiety in palliative care patients: Results of a pilot study. Complementary Therapies in Clinical Practice. 2006; 12(2):148-55. [DOI:10.1016/j. ctcp.2005.11.003] [PMID]

[12] Afshar M, Sattari Fard H, Shadi M, Ghaderi R. [Repairing effects of Iran flora on wound healing (Persian)]. Journal of Birjand University of Medical Sciences. 2015; 22(1):1-18. http://journal.bums.ac.ir/article1-1788-en.htm

[13] Boskabady MH, Shafei MN, Saberi Z, Amini S. Pharmacological effects of Rosa damascena. Iranian Journal of Basic Medical Sciences. 2011; 14(4):295-307. [PMID] [PMCID]

[14] Hamdamian S, Nazarpour S, Simbar M, Hajian S, Mojab F, Talebi A. Effects of aromatherapy with Rosa damascena on nulliparous women's pain and anxiety of labor during first stage of labor. Journal of Integrative Medicine. 2018; 16(2):120-5. [DOI:10.1016/j.joim.2018.02.005] [PMID]
[15] Marofi M, Sirousfard M, Moeini M, Ghanadi A. Evaluation of the effect of aromatherapy with Rosa damascena Mill. on postoperative pain intensity in hospitalized children in selected hospitals affiliated to Isfahan University of Medical Sciences in 2013: A randomized clinical trial. Iranian Journal of Nursing and Midwifery Research. 2015; 20(2):247-54. [PMID] [PMCID]

[16] Abbasi Maleki N, Abbasi Maleki S, Bekhradi R. Suppressive effects of Rosa damascena essential oil on naloxone-precipitated morphine withdrawal signs in male mice. Iranian Journal of Pharmaceutical Research. 2013; 12(3):357-61. [PMID] [PMCID]

[17] Nazıroğlu M, Kozlu S, Yorgancıgil E, Uğuz AC, Karakuş K. Rose oil (from Rosa $\times$ damascena Mill.) vapor attenuates depression-induced oxidative toxicity in rat brain. Journal of Natural Medicines. 2013; 67(1):152-8. [DOI:10.1007/s11418-012-0666-7] [PMID]

[18] Mohebitabar S, Shirazi M, Bioos S, Rahimi R, Malekshahi F, Nejatbakhsh $F$. Therapeutic efficacy of rose oil: A comprehensive review of clinical evidence. Avicenna Journal of Phytomedicine. 2017; 7(3):20613. [PMID] [PMCID]

[19] Kheirkhah M, Vali Pour NS, Nisani L, Haghani H. Comparing the effects of aromatherapy with rose oils and warm foot bath on anxiety in the first stage of labor in nulliparous women. Iranian Red Crescent Medical Journal. 2014; 16(9):e14455. [DOI:10.5812/ircmj.14455] [PMID] [PMCID]

[20] Niazi M, Hashempur MH, Taghizadeh M, Heydari M, Shariat A. Efficacy of topical Rose (Rosa damascena Mill.) oil for migraine headache: A randomized double-blinded placebo-controlled cross-over trial. Complementary Therapies in Medicine. 2017; 34:35-41. [DOI:10.1016/j. ctim.2017.07.009] [PMID]

[21] Davari M, Reihani M, Khoshrang N. [The aromatherapy effect of rosemary and lavander on primary dysmenorrhea: A clinical controlled trial (Persian)]. Journal of Isfahan Medical School. 2014; 32(290):929-37. http://jims.mui.ac.ir/index.php/jims/article/view/3157

[22] Najaf Najafi M, Hadavi F, Vazirinasab Kermani Sh, Vafisani F, Ghazanfarpour M. Aromatherapy with Iranian herbal medicines for premenstrual syndrome and primary dysmenorrhea: A systematic review and meta-analysis. International Journal of Pediatrics. 2019; 7(9):10155-66. [DOI:10.22038/IJP.2019.42455.3563]

[23] Tahmasbi H, Mahmoodi Gh, Mokhberi V, Hassani S, Akbarzadeh H, Rahnamai N. The impact of aromatherapy on the anxiety of patients experiencing coronary angiography. Zahedan Journal of Research in Medical Sciences. 2012; 14(3):51-5. https://www.researchgate.net/ publication/278001811

[24] Ayik C, Özden D. The effects of preoperative aromatherapy massage on anxiety and sleep quality of colorectal surgery patients: A randomized controlled study. Complementary Therapies in Medicine. 2018, 36:93-9. [DOI:10.1016/j.ctim.2017.12.002] [PMID]

[25] Moradi Kh, Ashtarian H, Darabi F, Hashemian AH, Saifi F. [A survey on the effects of Lavender aromatherapy on the anxiety and vital signs of patients with ischemic heart diseases hospitalized in cardiac intensive care units (Persian)]. Journal of Clinical Research in Paramedical Sciences. 2016; 4(4):301-10. http://5.63.15.58/attachmentsjson/download. action?masterCode $=17354$

[26] Nikfarjam M, Bahmani M, Heidari-Soureshjani S. Phytotherapy for anxiety in Iran: A review of the most important anti-anxiety medicinal plants. Journal of Chemical and Pharmaceutical Sciences. 2016; 9(3):1235-41. http://eprints.skums.ac.ir/933/

[27] Heidari-Fard S, Amir Ali-Akbari S, Rafiei A, Mojab F, Shakeri N, Simbar $M$. Investigating the effect of chamomile essential oil on reducinganxiety in nulliparous women during the first stage of childbirth. Internation- 
al Journal of Biology, Pharmacy and Allied Sciences. 2017; 6(5):828-42. https://ijbpas.com/archive/archive-single-pdf/2634

[28] Boswell MV, Eliot Cole B, editors. Weiner's pain management: A practical guide for clinicians. $7^{\text {th }}$ ed. Boca Raton: CRC Press; 2005. https:// books.google.com/books?id=E4aiDwAAQBAJ\&dq

[29] Babashahi M, Fayazi S, Mardanian Dehkordi L. [Comparing the effect of massage and inhalation aromatherapy on anxiety level of the patients in the preoperative period (Persian)]. Nursing and Midwifery Journal. 2015; 13(4):284-91. http://unmf.umsu.ac.ir/article-1-1432-en.html

[30] Zafarnia N, Kohan S, Abbaszadeh A, Nakhaei N, Miri S, Soleimani L. [The Effect of the therapeutic touch on preoperative anxiety in women with elective surgeries (Persian)]. Journal of Qualitative Research in Health Sciences. 2010; 10(1):42-51. http://jqr.kmu.ac.ir/ article-1-16-en.html

[31] Zakerimoghadam M, Aliasgharpoor M, Mehran A, Mohammadi S. [Effect of patient education about pain control on patients' anxiety prior to abdominal surgery (Persian)]. Journal of Hayat. 2010; 15(4):13-22. http://hayat.tums.ac.ir/article-1-101-en.html

[32] Kahangi LS, Moeini M, Babashahi M. [The effects of reflexology on anxiety levels before coronary artery bypass graft surgery (Persian)]. Journal of Research in Behavioural Sciences. 2012; 9(5):389-96. http://rbs.mui. ac.ir/index.php/jrbs/article/view/268

[33] Dagli R, Avcu M, Metin M, Kiymaz S, Ciftci H. The effects of aromatherapy using rose oil (Rosa damascena Mill.) on preoperative anxiety: A prospective randomized clinical trial. European Journal of Integrative Medicine. 2019; 26:37-42. [DOI:10.1016/j.eujim.2019.01.006]

[34] Fazlollahpour-Rokni F, Shorofi SA, Mousavinasab N, Ghafari R, Esmaeili $R$. The effect of inhalation aromatherapy with rose essential oil on the anxiety of patients undergoing coronary artery bypass graft surgery. Complementary Therapies in Clinical Practice. 2019; 34:201-7. [DOI:10.1016/j.ctcp.2018.11.014] [PMID] 
This Page Intentionally Left Blank 